Journal for ImmunoTherapy of Cancer

\title{
Development and validation of the immune signature to predict distant metastasis in patients with nasopharyngeal carcinoma
}

\author{
Sai-Lan Liu, ${ }^{1,2}$ Li-Juan Bian, ${ }^{3}$ Ze-Xian Liu, ${ }^{1}$ Qiu-Yan Chen, ${ }^{1,2}$ Xue-Song Sun, ${ }^{1,2}$ \\ Rui Sun, ${ }^{1,2}$ Dong-Hua Luo, ${ }^{1,2}$ Xiao-Yun Li, ${ }^{1,2}$ Bei-Bei Xiao, ${ }^{1,2}$ Jin-Jie Yan, ${ }^{1,2}$ \\ Zi-Jian Lu, ${ }^{1,2}$ Shu-Mei Yan, ${ }^{1,4}$ Li Yuan, ${ }^{1,2}$ Lin-Quan Tang, ${ }^{1,2}$ Jian-Ming Li, ${ }^{3}$ \\ Hai-Qiang Mai (i) 1,2
}

To cite: Liu S-L, Bian L-J, Liu Z-X, et al. Development and validation of the immune signature to predict distant metastasis in patients with nasopharyngeal carcinoma. Journal for ImmunoTherapy of Cancer 2020;8:e000205. doi:10.1136/jitc-2019-000205

- Additional material is published online only. To view please visit the journal online (http://dx.doi.org/10.1136/jitc2019-000205).

S-LL, L-JB, Z-XL, Q-YC and $X$-SS contributed equally.

L-QT, J-ML and H-QM are joint senior authors.

Accepted 09 March 2020

Check for updates

(c) Author(s) (or their employer(s)) 2020. Re-use permitted under CC BY-NC. No commercial re-use. See rights and permissions. Published by BMJ.

For numbered affiliations see end of article.

Correspondence to

Dr Hai-Qiang Mai;

maihq@mail.sysu.edu.cn

\section{ABSTRACT}

Background The tumor immune microenvironment has clinicopathological significance in predicting prognosis and therapeutic efficacy. We aimed to develop an immune signature to predict distant metastasis in patients with nasopharyngeal carcinoma (NPC).

Methods Using multiplexed quantitative fluorescence, we detected 17 immune biomarkers in a primary screening cohort of 54 NPC tissues presenting with/without distant metastasis following radical therapy. The LASSO (least absolute shrinkage and selection operator) logistic regression model used statistically significant survival markers in the training cohort ( $n=194)$ to build an immune signature. The prognostic and predictive accuracy of it was validated in an external independent group of 304 patients. Results Eight statistically significant markers were identified in the screening cohort. The immune signature consisting of four immune markers (PD-L1+ CD163+, CXCR5, CD117) in intratumor was adopted to classify patients into high and low risk in the training cohort and it showed a high level of reproducibility between different batches of samples ( $r=0.988$ for intratumor; $p<0.0001$ ). High-risk patients had shorter distant metastasis-free survival (HR 5.608, 95\% Cl 2.619 to $12.006 ; \mathrm{p}<0.0001$ ) and progression-free survival (HR 2.798, 95\% Cl 1.498 to 5.266; $\mathrm{p}=0.001$ ). The $\mathrm{C}$-indexes which reflected the predictive capacity in training and validation cohort were 0.703 and 0.636 , respectively. Low-risk patients benefited from induction chemotherapy plus concurrent chemoradiotherapy (IC+CCRT) (HR 0.355, 95\% Cl 0.147 to $0.857 ; p=0.021$ ), while high-risk patients did not (HR $1.329,95 \%$ Cl 0.543 to $3.253 ; p=0.533$ ). To predict the individual risk of distant metastasis, nomograms with the integration of both immune signature and clinicopathological risk factors were developed. Conclusions The immune signature provided a reliable estimate of distant metastasis risk in patients with NPC and might be applied to identify the cohort which benefit from IC+CCRT.

\section{INTRODUCTION}

In South China, nasopharyngeal carcinoma (NPC) is one of the most prevalent cancers. ${ }^{12}$
Of the 87000 newly diagnosed cases of NPC each year, more than $70 \%$ are locoregionally advanced disease. ${ }^{3}$ The prevention of distant metastasis, which is the main reason for treatment failure in advanced NPC remains unsatisfactory. ${ }^{4}$ The currently applied method for guiding treatment and predicting prognosis is mainly based on the tumor-node-metastases (TNM) staging system, which only take anatomical data into consideration and is insufficient to predict distant metastasis. Therefore, more precise diagnostic measures and effective treatments are required to guide individual treatment for patients with NPC.

Emerging evidence demonstrates that the specific tumor microenvironment could promote tumor progression and the diversity of its characteristics could be used for molecular classification, prediction of treatment responses and prognosis in a variety of cancers. ${ }^{5-7}$ NPC is characterized by abundant immunocell infiltration in the primary tumor, including $\mathrm{T}$ cells, B cells, mast cells (MCs), macrophages, and neutrophils. ${ }^{8}$ Previous studies suggested the adverse effects of the increase in macrophage, MCs, and neutrophils infiltration, which could play important roles in tumor support. ${ }^{9-11}$ In the meantime, many studies have reported that non-malignant lymphocytes infiltrating into the tumor and stroma was associated with favorable prognostic effects. ${ }^{12}{ }^{13}$ However, the antitumor response of these lymphocytes was often suppressed by immune checkpoints. Among them, programmed cell death-1 (PD-1) and T-cell immunoglobulin and mucin domain-containing protein 3 (TIM-3) were well-known immune checkpoints that hindered the function of activated $\mathrm{T}$ cells by mainly connecting programmed death-ligand 
1 (PD-L1) and galectin-9, respectively. ${ }^{14}$ In addition, lymphocyte-activation gene 3 (LAG-3) and galectin-1 were novel tumor immunotherapeutic targets, which recently attracted enormous attention. ${ }^{1617}$ These immune cells and immune checkpoints might serve as important identifiers permitting early diagnosis and subgroup classification. ${ }^{18} 19$ The previous studies on immune microenvironment of NPC were mostly presented with small sample size and an absence of independent validation, and mainly focused on limited number of markers. Besides, the coexpression of markers and the characteristics of non-malignant cells were often neglected. Since both malignant cells and stromal cells orchestrate in tumor-associated inflammation, tumor progression, and metastasis, ${ }^{20}$ it is of great necessity to picture the whole landscape of tumor immune microenvironment and pay attention to their relative locations. Furthermore, the difference in immune patterns among patients with various clinical outcomes, particularly distant metastasis should also be concerned and emphasized.

Cell-specific and topological analysis of immune checkpoint expression and immune cells in patients has become feasible with the development of fluorescent multiplex immunohistochemistry (IHC) and automated quantitation technology, which offers more objective perspectives and provides better prognostic information compared with conventional IHC-based pathological estimation. ${ }^{21} 22$ In the present study, a MultiOmyx platform was used to perform coexpression analysis and phenotype identification by integrating results from individual markers. In that case, we could simultaneously quantify the expression of 17 immune markers in intratumor and stromal tissues, including 6 important immune checkpoint molecules (PD-1, PD-L1, TIM-3, galectin-9, LAG-3, and galectin-1), and 11 relevant prognostic leukocyte markers: mature T lymphocytes (CD3), helper T cells (CD4), cytotoxic T cells (CD8), neutrophils (CD66b), T-cell follicular helper cells (CXCR5), regulatory T cells (Tregs) Foxp3+, monocytes (CD68), M2 macrophages (CD163), type 1 helper T cells (T-bet), and MCs (CD117), in a large cohort of NPC cases. The prediction of distant metastasis in patients with NPC can be further achieved by identifying and validating the immune signature. Moreover, we produced more accurate nomogram models for distant metastasis by integrating the immune signature with other clinical risk factors.

\section{PATIENTS AND METHODS Study population}

The study workflow is shown in figure 1 . The present study used the following eligibility criteria ${ }^{1}$ : newly diagnosed stage II-IVa NPC; ; did not receive any antitumor therapy before biopsy sampling ${ }^{3}$; received radical intensity modulated radiotherapy (IMRT) with or without chemotherapy $^{4}$; age $\geq 18$ years; Eastern Cooperative
A

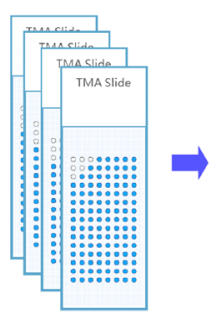

TMA Slides

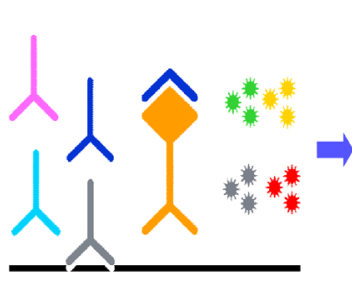

Multiplexed TSA staining

B

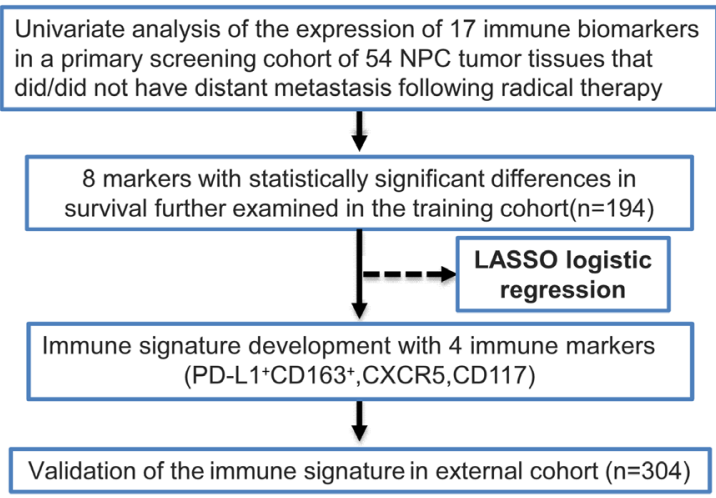

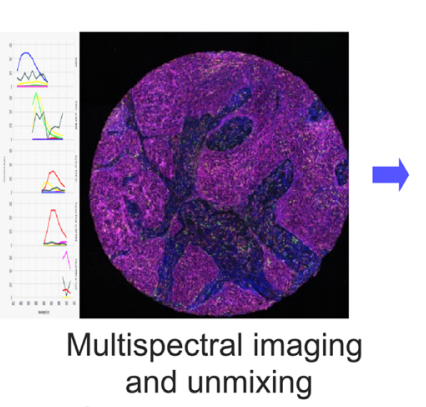

C

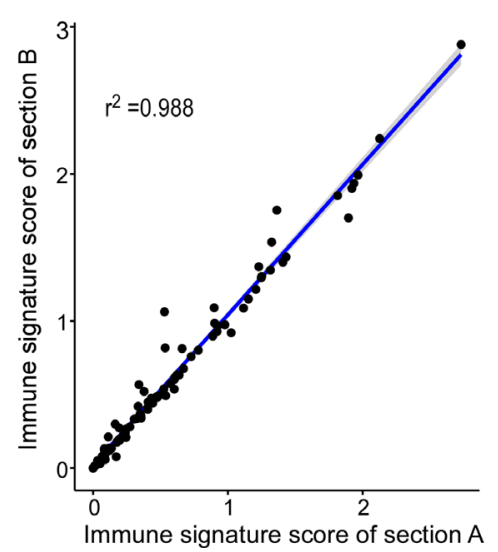

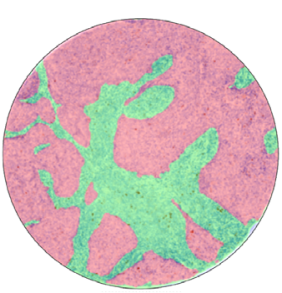

Tissue and cell segmentation

D

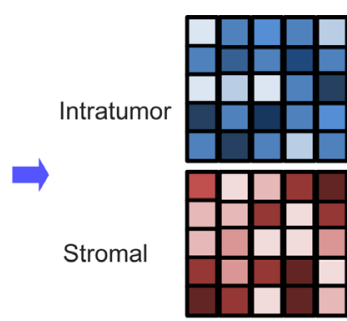

Phenotype Quantitation

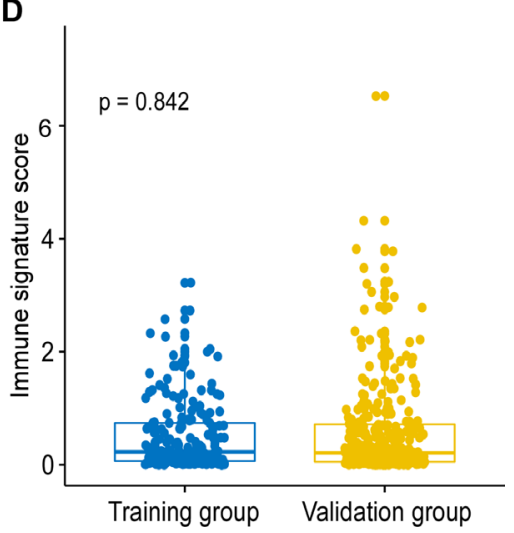

Figure 1 Workflow of the present study. (A) Process of multiplexed immunofluorescence staining and image analysis. (B) Study flow. (C) Example of a $2 \times 2$ correlation of the immune signature in the intratumor between two continuous sections of TMAs $(r=0.988)$. (D) Scatter diagram illustrating the immune signature A of the training and validation cohorts. Statistical comparison was performed by first testing normality using the Kolmogorov-Smirnov test, and then the Mann-Whitney non-parametric test was used to compare the two groups. LASSO, least absolute shrinkage and selection operator; NPC, nasopharyngeal carcinoma; TMA, tissue microarray; TSA, tyramide signal amplification. 
Oncology Group (ECOG) score between 0 and $2^{5}$; adequate hematological, renal, and hepatic functions; $\operatorname{and}^{6}$ no concomitant pregnancy, lactation, and other malignant disease. Between October 20, 2010 and March 16, 2016, 194 samples were obtained from patients treated at the Sun Yat-sen University Cancer Center (Guangzhou, China). As the external validation cohort, 304 samples were obtained from the Sun Yat-sen Memorial Hospital (Guangzhou, China) between December 12, 2011 and September 1, 2015. The eighth edition of the American Joint Committee on Cancer Staging Manual was used to restage all the patients. Theonline supplementary file 6(p1) showed detailed information concerning the radiotherapy dose and chemotherapy regimens. The plasma Epstein-Barr virus (EBV) DNA concentrations of patients were measured by quantitative PCR as described in the online supplementary file $6(\mathrm{p} 1,2)$.

\section{Multiplexed immunofluorescence staining}

One $1.0 \mathrm{~mm}$ tissue core from intratumor area was used to construct the tissue microarrays (TMAs), and another selected tissue core was available to locate stromal regions if possible in training cohort and one $1.0 \mathrm{~mm}$ tissue core from intratumor area was used to construct the TMAs in validation cohort. All tissue cores were reassessed by two pathologists (L-JB and S-MY), and tissue cores containing more than $70 \%$ tumor cells or stromal cells were included for further analysis, so the total area of intratumor and stromal tissue analyzed in each case was more than $0.55 \mathrm{~mm}^{2}$. All immunofluorescence staining was carried out on 4 - $\mu$ m-thick formalin-fixed, paraffinembedded TMAs. In the present study, we selected 17 prognostic markers mentioned earlier in this article for immunofluorescence staining of NPC tissues according to their involvement in cancer prognosis. ${ }^{9}{ }^{13} 23-30$ The biomarkers' names, antibody dilutions, and antibody clones are presented in online supplementary table 1 . online supplementary file 6 ( $\mathrm{p} 2,3$ ) detailed the method about validation and standardization of antibodies. PANO 7-plex IHC kit, cat 0004100100 (Panovue, Beijing, China) was used according to the manufacturer's protocol. Briefly, TMAs embedded in paraffin were deparaffinized, rehydrated, and subjected to antigen retrieval buffer treatment. Antigen retrieval was performed by microwave treatment in basic (EDTA, pH 9.0) antigen retrieval buffer. To block the endogenous peroxide activity, the sections were incubated in 3\% hydrogen peroxide for $10 \mathrm{~min}$ at room temperature, followed by washing in $1 \times$ Tris-buffered saline-Tween 20 . The sections were then incubated with each primary antibody separately for $60 \mathrm{~min}$. After washing, the sections were incubated for 10 min with a rabbit or mouse probe antibody specific for the species of the primary antibody, washed, and then incubated with a rabbit or mouse horseradish peroxidase conjugated secondary antibody for a further $10 \mathrm{~min}$. The sections were then washed and incubated with opal fluorophores (1:100 dilution in tyramide signal amplification reagent from the opal kit). For each additional marker, the protocol was repeated by treating the slides with an antigen retrieval step, followed by primary antibody staining and the subsequent downstream steps. Finally, all sections were stained using 2-(4-amidinophenyl)-1H-ind ole-6-carboxamidine (D9542, Sigma-Aldrich) for $3 \mathrm{~min}$. The sections were imaged under a fluorescent microscope fitted with an automated quantitative pathology imaging system called Polaris System (PerkinElmer, Waltham, Massachusetts, USA). Images were unmixed and annotated using inForm image analysis software (V.2.4, PerkinElmer). A combination of the percentage and intensity of positively stained cells was used to score the immune markers to generate a histochemistry $(\mathrm{H})$-score. ${ }^{31}$ online supplementary file 6 ( p 3-5) detailed the process of identification of intratumor and stromal tissue and the multispectral imaging and scoring standard for the immune markers.

\section{Statistical analysis}

The H-score or percentage of immune cells obtained from all available cores in the intratumor and stromal tissues of each case were used. We first compared 54 patients with patients with NPC at the Sun Yat-sen University Cancer Center with metastasis $(n=25)$ or without metastasis $(n=29)$ after radical therapy as the original training group. This group was well balanced in terms of T stage, gender, age, $\mathrm{N}$ stage, and treatment method, which eliminated the effect of these factors on metastasis (online supplementary table 2). Univariate analyses using Cox proportional hazards regression modeling was then used to test the significance of different immune markers in this original training group. The $\mathrm{p}$-value for significant markers $(<0.05)$ remained for further validation within the training group.

To construct a prediction model in the training group, we used a penalized logistic model to select markers. The coefficients weighted by the penalized logistic model were used to construct the prediction model using the $\mathrm{R}$ package glmnet in the training cohort (online supplementary file 6 (p 5) ). In the training cohort, the X-tile software (V.3.6.1; Yale University, New Haven, Connecticut, USA), which can automatically select the optimum data threshold according to the highest $\chi^{2}$ value (minimum $p$ value) defined by Kaplan-Meier survival analysis and logrank test ${ }^{32}$ was used to identify the optimal cut-off value.

Distant metastasis-free survival (DMFS) was the primary endpoint, defined as the interval between the first day of diagnosis to the advent of first distant metastasis event. Progression-free survival (PFS) was the secondary endpoint, defined as the interval between the first date of diagnosis and disease progression or death from any cause. The Kaplan-Meier method was used to analyze survival, and the log-rank test was used to compare the differences between groups. We calculated the HRs using univariate Cox regression analysis. As a predictor of benefit gained from induction chemotherapy plus concurrent chemoradiotherapy (IC+CCRT), the immune 
signature was analyzed in the combined training and external cohorts.

Multivariate Cox regression analysis with backward selection was performed to test the independent significance of different variables. To remove non-significant variables from the analysis, the threshold $p$ value was set at 0.1 ( $p>0.1$ ), and the final Cox model retained marginally significant variables $(005<\mathrm{p}<0.1)$. The following covariates were involved in the model, including the immune signature (high risk vs low risk), gender (male vs female), age ( $\geq 45$ years vs $<45$ years), T stage (T3-4 vs T1-2), $\mathrm{N}$ stage (N2-3 vs N0-1), ECOG (0 vs 1 vs 2 ), serum lactate dehydrogenase ( $\geq 245 \mathrm{vs}<245 \mathrm{U} / \mathrm{L})$, C reactive protein ( $\geq 3.0 \mathrm{vs}<3.0 \mathrm{mg} / \mathrm{L}$ ), hemoglobin (HGB; $<130 \mathrm{vs} \geq 130 \mathrm{~g} / \mathrm{L}$ ) and body mass index ( $\left.\geq 23 \mathrm{vs}<23 \mathrm{~kg} / \mathrm{m}^{2}\right)$. For the multivariable Cox regression model, the coefficients were used to construct nomograms. The calibration curves were made by plotting the observed rates against the predicted probabilities of nomogram. A bootstrapping method with 1000 resamples was used to calculate the concordance index (C-index). To evaluate the prognostic or predictive accuracy of the clinical features and the immune signature-based nomogram, receiver operating characteristic (ROC) analysis was used. The area under the ROC curve was calculated to assess the sensitivity and specificity of the model to predict distant metastasis. All statistical analyses were performed using R (V.3.6.0) and SPSS (V.22.0, IBM). The statistical tests were all two-sided, and statistical significance was indicated by a p-value of less than 0.05 .

\section{RESULTS \\ Patient characteristics}

Table 1 summarized the patient characteristics in the training cohort $(\mathrm{n}=194)$ and external validation cohort $(\mathrm{n}=304)$. The patients had a median age of 46 years (range: 18-78) years. There were $150(30.1 \%$ ) female patients and $348(69.9 \%)$ male patients. Seventy-five (15.1\%) patients were diagnosed with stage II disease, 252 $(50 \cdot 6 \%)$ with stage III, and $171(34.3 \%)$ with stage IVa. All 498 patients underwent radical IMRT. Additionally, among them, $201(40.4 \%)$ received IC plus CCRT and $187(37.6 \%)$ received CCRT alone. The median follow-up was 62.0 months (IQR 26.1-60.2), and 62 (12.4\%) of the 498 patients developed distant metastasis during the follow-up period.

\section{Screening results of the primary training group}

Data for 54 intratumor cores and 40 stromal cores containing more than $70 \%$ tumor cells or stromal cells from 54 specimens of the primary training cohort were used for further analysis. We used the median $\mathrm{H}$-score or percentage as a cut-off score and found that the expression of LAG-3 and CD117 in the intratumor region were individually associated with DMFS. In addition, the ratio of $\mathrm{TIM}^{-} 3^{+} \mathrm{CD}^{+}{ }^{+} \mathrm{T}$ cells and PD-L1 ${ }^{+} \mathrm{CD} 163^{+}$cells yielded strong negative associations with DMFS and the ratio of
PD- $1^{+} \mathrm{CD} 8^{+} \mathrm{T}$ cells and $\mathrm{CD} 39^{+} \mathrm{CD} 8^{+}$cells had no significant correlation with DMFS. As regard to stromal cells, high expression of CXCR5 and Foxp3 were identified with poorer DMFS (online supplementary table 3). The above eight significant immune markers (TIM-3, CD8, PD-L1, CD163, LAG-3, CXCR5, Foxp3, and CD117) were adopted for further verification using the training cohort of 194 patients. The eight selected immune markers showed distinguishable and clear staining as shown in online supplementary figure 1 .

\section{Association of the immune signature with prognosis}

The least absolute shrinkage and selection operator (LASSO) logistic regression model was used to build prognostic immune signature $\mathrm{A}$, which involved four markers in intratumor (percentage of PD-L1 ${ }^{+} \mathrm{CD} 163^{+}$, CXCR5, CD117) identified by the training set $(n=194)$ (online supplementary figure 2). The immune signature of each patient was calculated based on their regression coefficients of the expression levels of these immune markers in the intratumor (online supplementary table 4): Immune signature $\mathrm{A}=\left(0.013479 \times 1000 \times\right.$ percentage of $\mathrm{PD}-\mathrm{L} 1^{+}$ CD163 $\left.{ }^{+}\right)+(0.057512 \times \mathrm{H}$-score of CXCR5 $)+(0.011048 \times \mathrm{H}-$ score of CD117). Immunofluorescence staining was carried out on two continuous sections of TMAs and it showed strong repeatability and reproducibility between different batches $(r=0.988$ for intratumor; $\mathrm{p}<0.0001$; figure 1C). In the training cohort, the patients were separated into low-risk and high-risk groups using an optimal cut-off value generated by X-tile plots (online supplementary figure 3 ). The low-risk and high-risk group, respectively, comprised $78.4 \%(152 / 194)$ and $21.6 \%(42 / 194)$ of the patients. The 5-year DMFS was $61.4 \%$ (95\% CI $45.9 \%$ to $76.9 \%)$ in the high-risk group and $89.1 \%$ (95\% CI $82.2 \%$ to $96.0 \%$ ) in the low-risk group (HR $5.608,95 \%$ CI 2.619 to $12.006 ; \mathrm{p}<0.0001$; figure 2 ; online supplementary table 5). Patients in the high-risk group also had shorter PFS values (HR 2.798, 95\% CI 1.498 to 5.226; $\mathrm{p}=0.001$; figure 2; online supplementary table 5) compared with that in the low-risk group.

Both malignant cells and stromal cells orchestrated in tumor progression and metastasis. Therefore, we developed immune signature B by taking both intratumor and stromal conditions into consideration and found no statistically significant difference between immune signature B and immune signature A (data not shown). Obtained by biopsy forceps, the NPC tissue was too tiny for every tissue section to contain enough stromal tissue to construct TMA and there were only $65.5 \%(127 / 194)$ of patients with available data for stromal cores in our study, which indicated a limited application value of immune signature B in clinical practice. Given the better accessibility and similar prognostic value of immune signature $\mathrm{A}$, it was selected to predict the prognosis of patients with NPC in validation cohort. A scatter diagram illustrating the immune signature of the training and validation cohorts was shown in figure 1D with no significant difference in immune signature distribution observed between the 
Table 1 Clinical characteristics of patients in the training and external validation cohorts

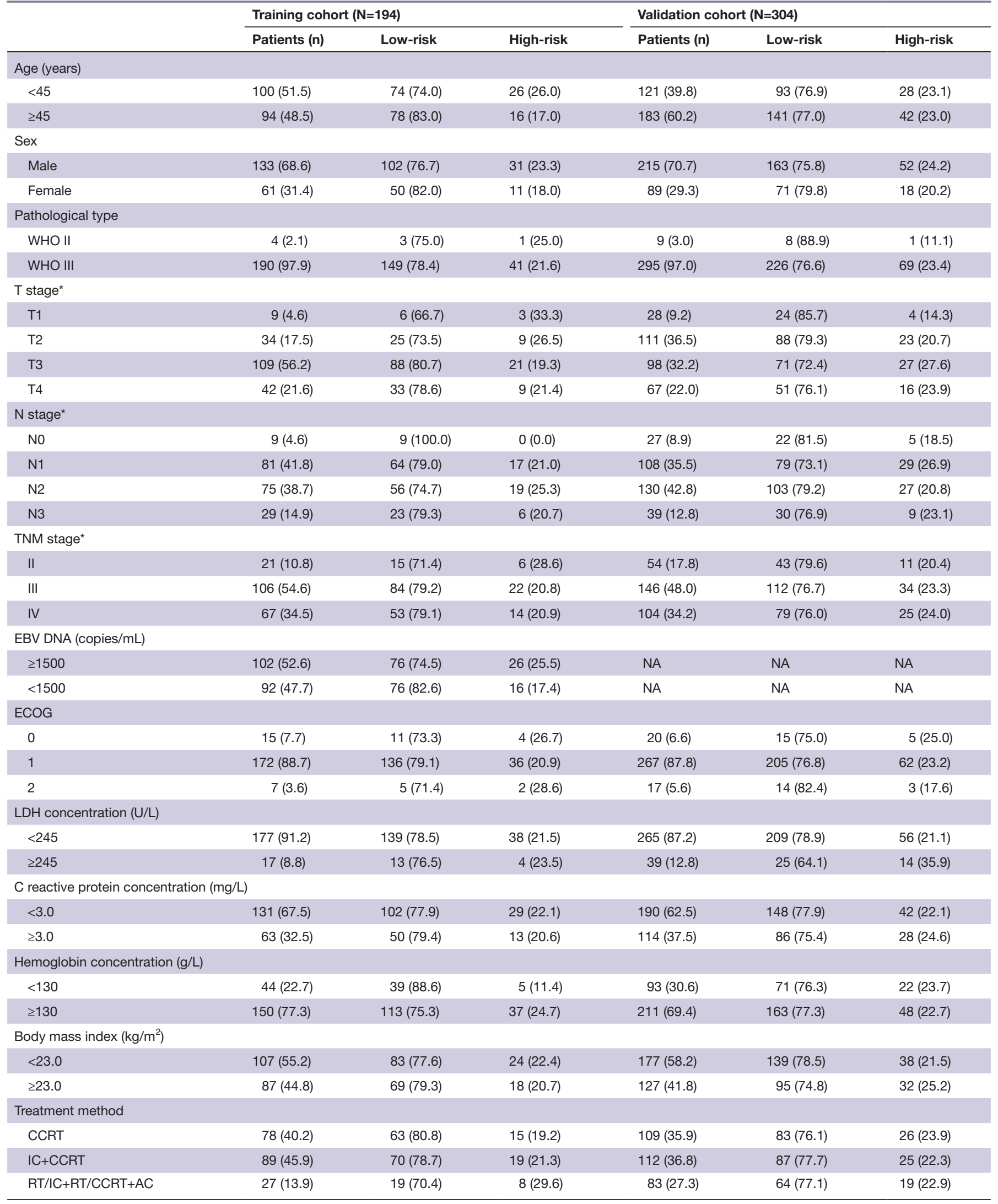

*According to the eighth edition of UICC/AJCC staging system.

AC, adjuvant chemotherapy; AJCC, American Joint Committee on Cancer; CCRT, concurrent chemoradiotherapy; EBV, Epstein-Barr virus; ECOG, Eastern Cooperative Oncology Group; IC, induction chemotherapy; LDH, serum lactate dehydrogenase; NA, not available; RT, radiotherapy; TNM, tumor-node-metastases; UICC, Union for International Cancer Control. 

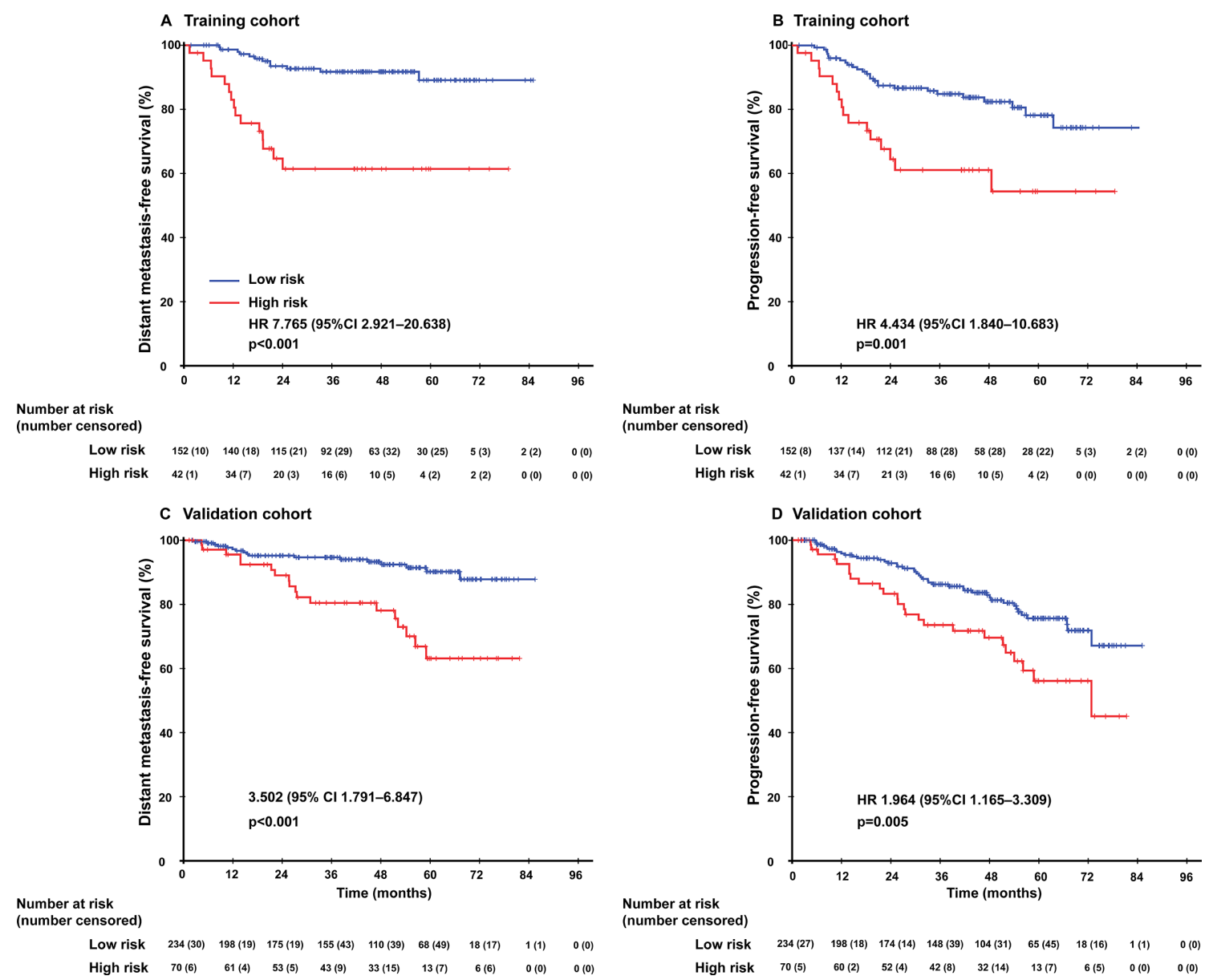

Figure 2 Kaplan-Meier curves for distant metastasis-free survival and progression-free survival between the immune signature-defined high-risk and low-risk groups in the training and validation cohorts.

two groups $(\mathrm{p}=0.842)$. In the validation cohort, immune signature A categorized $234(81.3 \%)$ of the 304 patients into low-risk group and 70 patients $(18.7 \%)$ into highrisk group. Patients in high-risk group demonstrated with far shorter DMFS (HR 3.502, 95\% CI 1.791 to 6.847 ; $\mathrm{p}<0.0001$; figure 2, online supplementary table S5) and PFS (HR 1.964, 95\% CI 1.165 to 3.309; $\mathrm{p}=0.011$; figure 2, online supplementary table S5) in contrary to low-risk patients.

\section{Benefit of induction chemotherapy}

Among the 498 patients in the combined cohort, 167 $(33.5 \%)$ received CCRT and $221(44.8 \%)$ received IC+CCRT. For these patients, survival outcomes were comparable between IC+CCRT and CCRT alone groups (figure 3). Then, the immune signature was applied to predict the survival benefit gained from IC. Stratification of immune signature A with Kaplan-Meier survival analysis showed that treatment with IC+CCRT rather than CCRT was associated with improved DMFS (HR $0.355,95 \%$ CI 0.147 to $0.857 ; \mathrm{p}=0.021$ ) and PFS (HR $0.590,95 \% \mathrm{CI} 0.351$ to $0.992 ; \mathrm{p}=0.047$ ) in patients with low immune signature scores rather than those with high immune signature scores (DMFS: HR 1.294, 95\% CI 0.553 to 3.029; $\mathrm{p}=0.552$; PFS: HR 1.248, 95\% CI 0.590 to 2.639 ; $\mathrm{p}=0.563$; figure 3 ).
Online supplementary table S6 in the appendix showed the 5-year DMFS and PFS in each risk group.

\section{Establishment and validation of nomograms with the immune signature}

After multivariable adjustment by clinicopathological risk factors, immune signature A remained a strong independent prognostic factor for DMFS in the training cohort (HR 6.295 , 95\% CI 2.886 to 13.729 ; $\mathrm{p}<0.0001$; table 2) and in the validation cohort (HR 4.297, 95\% CI 2.182 to 8.461; $\mathrm{p}<0.001$; table 2 ). In addition, $\mathrm{N}$ stage (HR 2.522, 95\% CI 1.086 to 5.857; $\mathrm{p}=0.031$ ) and HGB (HR 0.335, $95 \%$ CI 0.126 to $0.890 ; p=0.028$ ) also remained significant for DMFS after adjustment for various cofactors. Among them, immune signature A had the highest C-index (0.703, 95\% CI 0.613 to 0.793 ; C-index $0.636,95 \%$ CI 0.551 to 0.721 ) both in training and validation cohort (online supplementary table S7). Then, we constructed nomogram $\mathrm{A}$ to predict the 5-year DMFS by combining the variables of immune signature $\mathrm{A}, \mathrm{N}$ stage, and pretreatment HGB levels (figure 4). The calibration plots for the nomogram A were predicted well in the training cohort (C-index $0.791,95 \%$ CI 0.720 to 0.862 ) and the validation cohort (C-index $0.729,95 \%$ CI 0.630 to 0.828 ; figure 4 ). 
A Combined cohort

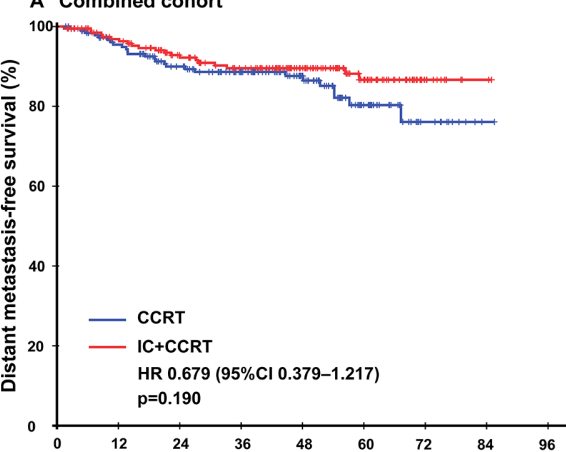
(number censored)

$\begin{array}{llllllllll}\text { CCRT } & 187(17) & 162(17) & 136(23) & 111(32) & 78(38) & 35(23) & 11(10) & 1(1) & 0(0)\end{array}$

$\begin{array}{llllllllll}\text { IC+CCRT } & 201(18) & 177(22) & 148(15) & 128(35) & 93(38) & 53(40) & 13(11) & 2(2) & 0(0)\end{array}$

C Low risk cohort

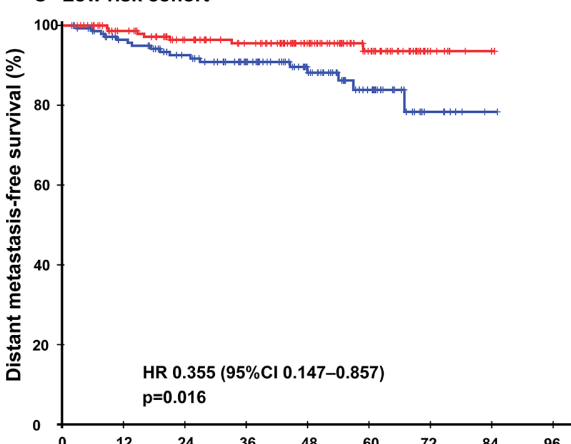

Number at risk

(number censored)

$\begin{array}{llllllllll}\text { CCRT } & 146(14) & 127(12) & 110(17) & 91(28) & 62(29) & 30(22) & 7(6) & 1(1) & 0(0)\end{array}$

$\begin{array}{llllllllll}\text { IC+CCRT } & 157(16) & 139(18) & 118(13) & 104(26) & 78(33) & 44(35) & 9(7) & 2(2) & 0(0)\end{array}$

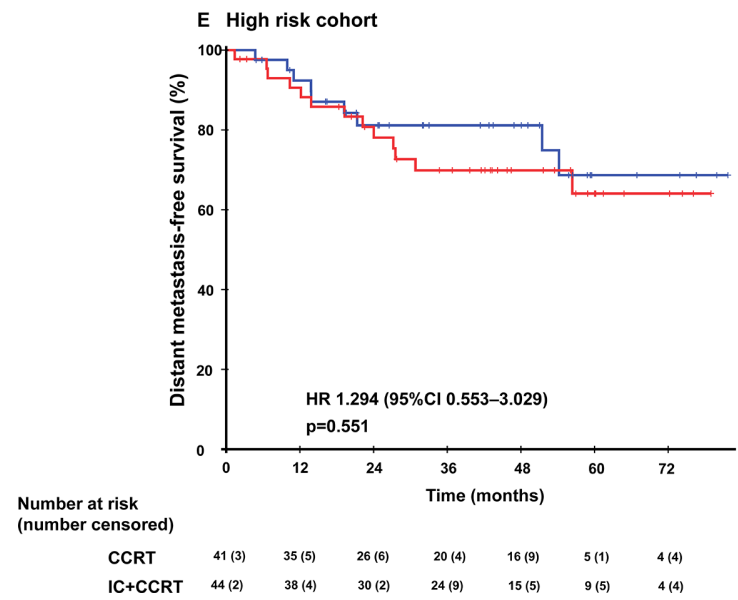

B Combined cohort

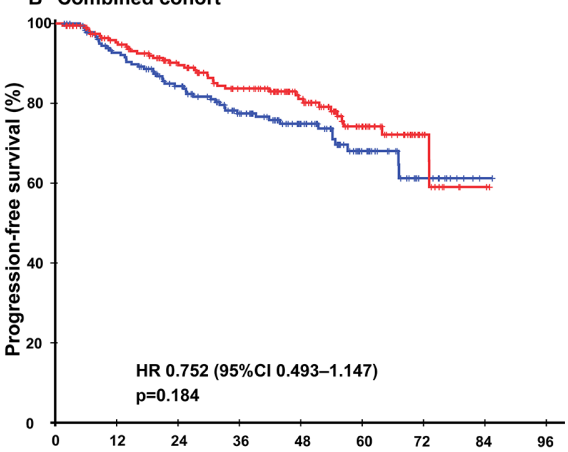

Number at risk

$\begin{array}{llllllllll}\text { CCRT } & 187(14) & 160(14) & 132(18) & 104(29) & 72(34) & 33(20) & 11(10) & 1(1) & 0(0)\end{array}$

$\begin{array}{llllllllll}\text { IC+CCRT } & 201(17) & 175(19) & 147(15) & 122(32) & 87(31) & 50(36) & 13(9) & 2(2) & 0(0)\end{array}$

D Low risk cohort

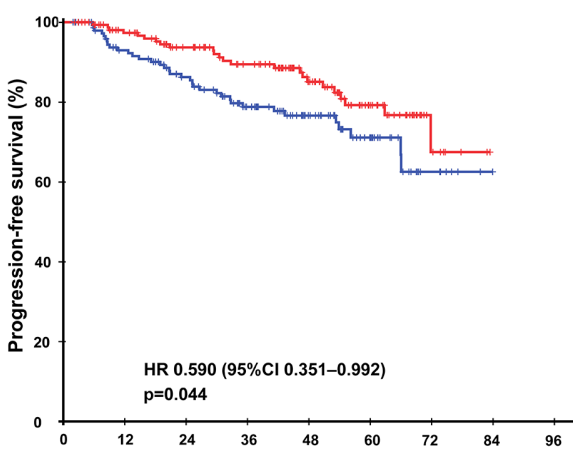

Number at risk (number censored)

$\begin{array}{llllllllll}\text { CCRT } & 146(11) & 125(10) & 106(13) & 84(26) & 56(25) & 28(19) & 7(6) & 1(1) & 0(0)\end{array}$

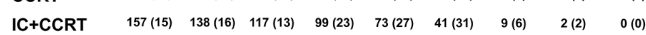

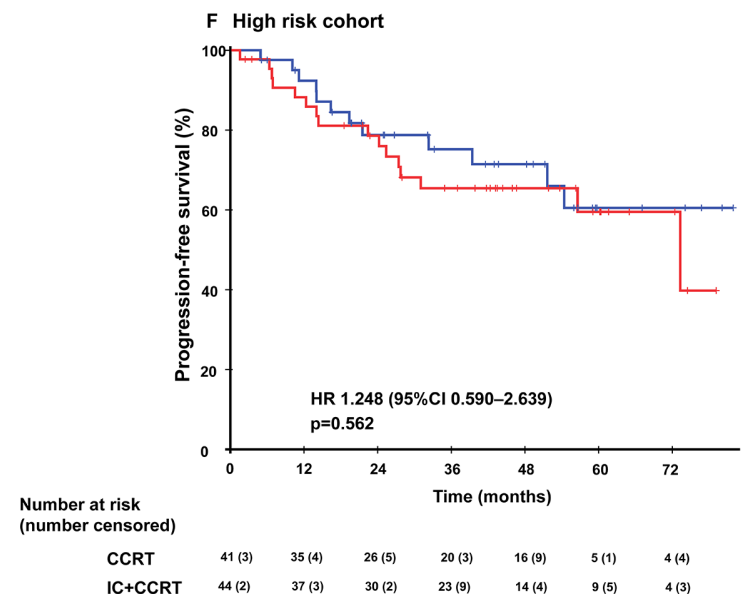

Figure 3 Kaplan-Meier survival curves between IC+CCRT and CCRT alone in different groups. Distant metastasis-free survival (A) and progression-free survival (B) for the whole combined cohort; distant metastasis-free survival (C) and progression-free survival (D) for the immune signature-defined low-risk patients in the combined cohort; distant metastasis-free survival (E) and progression-free survival $(F)$ for the immune signature-defined high-risk patients in the combined cohort. We calculated $p$ values using the unadjusted log-rank test and HRs using a univariate Cox regression analysis. CCRT, concurrentchemoradiotherapy; IC, induction chemotherapy.

Lin $e$ t $a p^{33}$ demonstrated that the prognosis of patients with NPC could be distinguished by EBV DNA levels with a cut-off point of 1500 copies/mL. Previous studies showed that plasma EBV DNA level was considered the most potential biomarker to complement the TNM stage,$^{34}$ and we developed a nomogram B on the basis of training cohort using plasma EBV DNA status, N stage, HGB, and immune signature A (online supplementary figure 4). Both nomogram A and nomogram B boosted higher predictive value compared with that of EBV DNA level or $\mathrm{N}$ category in the training cohort (online supplementary table S8). Nonetheless, the addition of EBV DNA 
Table 2 Multivariable Cox regression analysis of prognostic factors in the training cohort and validation cohort

\begin{tabular}{|c|c|c|}
\hline & $\mathrm{HR}^{*}(95 \% \mathrm{Cl})$ & $P$ value \\
\hline \multicolumn{3}{|l|}{ Distant metastasis-free survival } \\
\hline \multicolumn{3}{|l|}{ Training cohort $(n=194)$} \\
\hline HGB $(\geq 130$ vs $<130 \mathrm{~g} / \mathrm{L})$ & $0.335(0.126$ to 0.890$)$ & 0.028 \\
\hline$N$ category (2-3 vs $0-1)$ & $2.522(1.086$ to 5.857$)$ & 0.031 \\
\hline $\begin{array}{l}\text { Immune signature (high vs } \\
\text { low) }\end{array}$ & 6.295 (2.886 to 13.729$)$ & $<0.001$ \\
\hline \multicolumn{3}{|l|}{ Validation cohort $(n=304)$} \\
\hline Age ( $\geq 45$ years vs $<45$ years) & $3.003(1.355$ to 6.654$)$ & 0.007 \\
\hline $\mathrm{N}$ stage (N2-3 vs N0-1) & 3.461 (1.501 to 7.979$)$ & 0.004 \\
\hline $\begin{array}{l}\text { Immune signature (high vs } \\
\text { low) }\end{array}$ & 4.297 (2.182 to 8.461$)$ & $<0.001$ \\
\hline \multicolumn{3}{|l|}{ Progression-free survival } \\
\hline \multicolumn{3}{|l|}{ Training cohort $(n=194)$} \\
\hline $\mathrm{N}$ category (2-3 vs $0-1)$ & 2.136 (1.107 to 4.118$)$ & 0.024 \\
\hline $\begin{array}{l}\text { Immune signature (high vs } \\
\text { low) }\end{array}$ & 2.775 (1.484 to 5.189$)$ & 0.001 \\
\hline \multicolumn{3}{|l|}{ Validation cohort $(n=304)$} \\
\hline HGB $(\geq 130$ vs $<130 \mathrm{~g} / \mathrm{L})$ & 0.535 (0.325 to 0.878$)$ & 0.013 \\
\hline Age ( $\geq 45$ years vs $<45$ years) & $2.018(1.194$ to 3.411$)$ & 0.009 \\
\hline $\mathrm{N}$ stage (N2-3 vs N0-1) & 1.713 (1.028 to 2.854$)$ & 0.045 \\
\hline $\begin{array}{l}\text { Immune signature (high vs } \\
\text { low) }\end{array}$ & 2.115 (1.289 to 3.469$)$ & 0.003 \\
\hline
\end{tabular}

HRs and $p$ values were calculated using an adjusted multivariate Cox proportional hazards regression model, immune signature (high risk vs low risk), gender (male vs female), age ( $\geq 45$ years vs $<45$ years), $T$ stage (T3-4 vs T1-2), N stage (N2-3 vs N0-1), overall stage (I-III vs IV), ECOG ( 0 vs 1 vs 2 ), LDH ( $\geq 245$ vs $<245 \mathrm{U} / \mathrm{L})$, CRP ( $\geq 3 \mathrm{vs}<3 \mathrm{mg} / \mathrm{L})$, HGB $(\geq 130 \mathrm{vs}<130 \mathrm{~g} / \mathrm{L})$, and BMI ( $\left.\geq 23 \mathrm{vs}<23 \mathrm{~kg} / \mathrm{m}^{2}\right)$ were included as covariates. Variables were selected with the backward stepwise approach, and the $p$ value threshold was $0.1(p>0.1)$ for removing insignificant variables from the model. Only variables significantly associated with survival were presented, and marginally significant variables $(0.05<p<0.1)$ were remained in the final Cox model but not presented in the table.

$\mathrm{BMI}$, body mass index; CRP, serum $\mathrm{C}$ reactive protein; ECOG, Eastern Cooperative Oncology Group; HGB, hemoglobin; LDH, serum lactate dehydrogenase.

to nomogram B (C-index $0.805,95 \%$ CI 0.718 to 0.892 ) did not enhance the accuracy compared with nomogram A (C-index $0.791,95 \%$ CI 0.720 to $0.862 ; \mathrm{p}=0.386$ ) (online supplementary table S8). The EBV DNA data was unavailable in the validation cohort, thus it was used only to compare the sensitivity and specificity of the nomogram A with $\mathrm{N}$ category (online supplementary table S8).

\section{DISCUSSION}

By using fluorescent multiplex IHC and automated quantitation technology, which showed strong repeatability and reproducibility between different batches, this study developed and validated an objective immune signature tool, with four intratumor markers incorporated, the infiltration of CD163+ macrophage cells expressing PD-L1, and the expression of CD117 and CXCR5. The combination of them categorized patients into high risk and low risk with significantly different DMFS. In addition, patients identified as low risk by the immune signature could benefit more from IC+CCRT.

The prognostic value of PD-L1 in NPC remains controversial. Zhu et al reported that PD-L1 expression on tumor cells was associated with favorable prognosis in a subgroup of patients with NPC who exhibited pre-existing lymphocytes infiltrating in tumor. ${ }^{18}$ A study conducted by Fang demonstrated that PD-L1 was associated with worse disease-free survival in patients with NPC, ${ }^{35}$ while Chan reported the insufficient prognostic value of PD-L1 expression. ${ }^{36}$ The predictive effect of PD-L1 may be partly determined by the different expression of it in immune cells or tumor cells. ${ }^{37}$ In this study, we demonstrated that more expression of PD-L1 on intratumor CD163+macrophages was associated with distant metastasis, which was consistent with previous study. ${ }^{38}$ In recent years, PD-1 and PD-L1 therapies have shown remarkable clinical efficacy in numerous advanced solid cancers. ${ }^{39-41}$ However, response rate of anti-PD-1/PD-L1 treatment in patients with recurrent or metastatic NPC only ranged from $20 \%$ to $34 \%{ }^{42-44}$ It was crucial to identify effective indicators for predicting efficacy of PD-1 and PD-L1 therapies. Previous studies reported about the potential indispensable role of PD-L1 expressed by non-tumor cells on PD-L1 blockade-mediated tumor elimination in murine tumor models and the correlation between PD-L1-positive macrophages and the positive effects of anti-PD-L1 and anti-PD-1 antibodies in lung cancers and melanoma. ${ }^{45-47}$ Our study might offer a clue to select intratumor PD-L1positive macrophages as promising biomarker for the use of checkpoint inhibitors.

MCs migrated to the tumor microenvironment in response to stem cell factors secreted by tumor cells. Data suggested that $\mathrm{CD} 117^{+}$MCs promoted tumor progression by suppressing the immune system and also in several other ways. ${ }^{48}$ It has also been reported that increased MC number correlated with an adverse prognosis. ${ }^{49}$ Similar association between high CXCR5 expression and poorer prognosis was also observed in previous study in a variety of cancers. ${ }^{5051}$ Consistent with previous studies, our results showed that high expression of CD117, CXCR5 were associated with poorer prognosis in patients with NPC, which were worth to be considered as future antitumor targets.

Furthermore, our results showed that patients classified as low risk instead of high risk would benefit from IC+CCRT, that is, induction chemotherapy might not be effective enough for high-risk group to eradicate micrometastasis, and more aggressive therapeutic strategies should be considered in this cohort. Patients classified as high risk by the immune signature were believed to have abundant infiltrating immune cells along with elevated level of checkpoint markers, and that indicated that immunotherapy might work well in this population. In brief, the developed immune signature can be applied not only as a prognostic tool but also as a guidance for giving individualized antitumor treatment, such 


\section{A}

Points

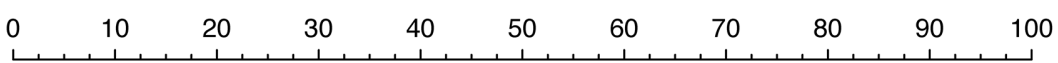

Immunoscore A

$$
\text { Low risk }
$$

High risk

$\mathrm{N}$ stage

HGB

$$
\text { No-1 } \quad \geq 130 \mathrm{~g} / \mathrm{L}
$$$$
<130 \mathrm{~g} / \mathrm{L}
$$

Total Points

\begin{tabular}{lllllllllllll}
\hline 0 & 20 & 40 & 60 & 80 & 100 & 120 & 140 & 160 & 180 & 200
\end{tabular}

\begin{tabular}{|c|c|c|}
\hline 0.95 & 0. & 0 \\
\hline
\end{tabular}

3-year DMFS

\begin{tabular}{|c|c|}
\hline 0.95 & \\
\hline
\end{tabular}

5-year DMFS

B

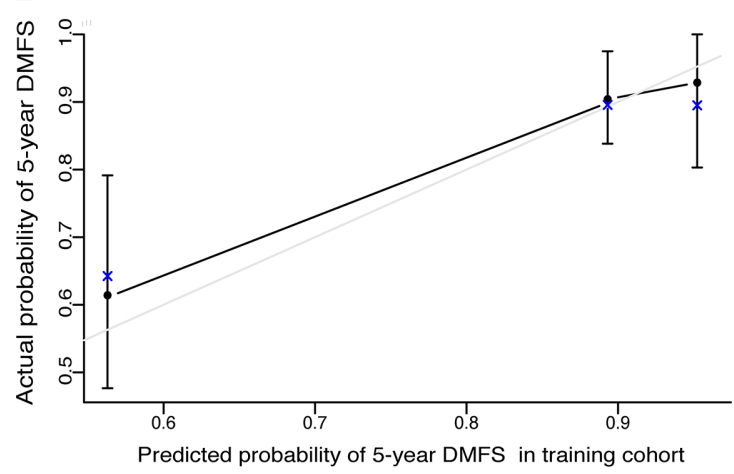

C

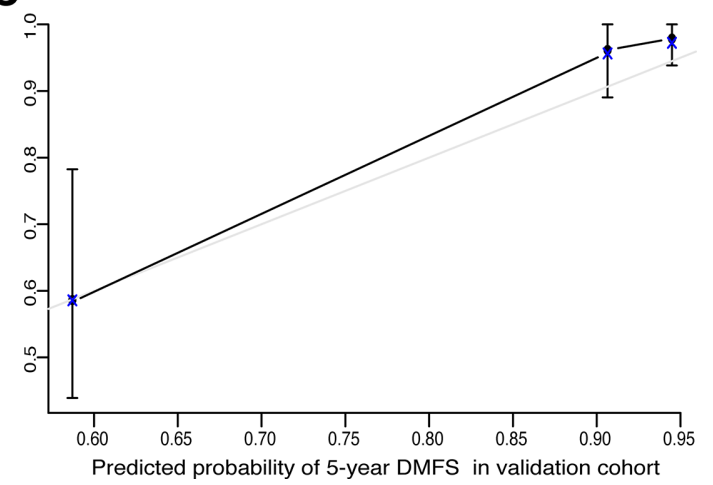

Figure 4 Nomogram A to predict the risk of distant metastasis in nasopharyngeal carcinoma (A). Calibration curves of the nomogram to predict DMFS at 5 years in: $(B)$ the training cohort and $(C)$ the external validation cohort. The actual DMFS was plotted on the $y$-axis and the nomogram predicted probability was plotted on the x-axis. HGB, hemoglobin; DMFS, distant metastasis-free survival.

as choosing appropriate candidates for induction chemotherapy. However, as the above views are yet theoretical conjecture, it is necessary to carry out prospective clinical trials to verify the applicability of our research results in clinical practice.

To provide a more accurate profile to predict the accurate risk of distant metastasis for each patient, nomograms were built. Nomogram A, with N stage, HGB, and immune signature $\mathrm{A}$ incorporated had an excellent predictive accuracy, and nomogram B was formed on the basis of nomogram A with the addition of EBV DNA level. However, the complement of EBV DNA to nomogram A did not enhance the accuracy of nomogram B, which may be explained by the existence of potential correlation between EBV and immune features, as it was reported that $69 \%$ EBV-related NPC exhibited high expression of PD-L1. ${ }^{52}$ On the other hand, since EBV DNA detection methods varied among institutes, the generalization of nomogram B including EBV DNA might be hindered. Thus, nomogram A, which showed same accuracy, was worth to be popularized for predicting prognosis for locally advanced NPC. In addition, we have also tried the LASSO Cox regression model to build the immune signature and found that only one marker in intratumor was selected (CD117), which had similar prognostic value with immune signature A built by LASSO logistic regression (ROC $0.711,95 \%$ CI 0.580 to 0.843 vs ROC $0.668,95 \%$ CI 0.478 to $0.858 ; \mathrm{p}=0.618$ ) concerning timeindependent 5-year ROC. In spite of the handy nature of one single marker in clinic, the combined immune signature built by LASSO logistic regression model (percentage of PD-L1+ CD163+, CXCR5, CD117) could reveal more information about immune status and offer insight into the immunotherapy of patients with NPC.

The present study had several limitations. First, the data were obtained in NPC-endemic area in China, and the pathological subtype was mainly undifferentiated nonkeratinizing carcinoma. The application of it in areas with distinct pathological subtypes and clinical characteristics should be revalidated. Second, the number of immune markers screened in the training cohort was limited, which resulted in a smaller panel of genes being integrated into the immune signature compared with other gene expression profiling studies such as complementary DNA arrays. Third, the number of fluorescent channels of the multiplexed immunofluorescence staining was limited by the 
overlap of fluorescent signals, thus all markers into one panel and their multiple combinations are difficult to put into practice. Fourth, the NPC tissue was obtained by biopsy forceps and it was hard to get the whole tumor. We evaluated the expression of each immune marker on single biopsy, which might not be representative enough to reflect the integrated immune pattern of patients with NPC because of the possible tumor heterogeneity. Fifth, confined to the sample size and follow-up duration, many patients who had tumor progression were still alive with tumor burden, thus the prediction efficiency for overall survival (OS) was not as good as those for distant metastasis and progression. Further studies with more patients and longer follow-up times would be useful to evaluate the impact of immune signature on OS. Furthermore, we did not explore the value of the immune signature as a predictive tool to identify patients who would benefit from immunotherapy in clinical practice. Therefore, further studies were encouraged to explore the value of the immune signature for identifying candidates for immunotherapy, and the validation by prospective studies from other NPC-endemic areas were also expected.

\section{CONCLUSION}

Our findings showed that the prognostic tool comprising a multiplexed quantitative fluorescence-based objective immune signature could effectively classify locoregionally advanced patients with NPC into different risk groups for distant metastasis. Moreover, we showed that patients identified as low risk using the developed immune signature tool could benefit more from IC+CCRT. The immune signature-derived nomogram could provide clinicians with practical and accessible information for predicting prognosis.

\section{Author affiliations \\ ${ }^{1}$ Sun Yat-sen University Cancer Center, State Key Laboratory of Oncology in South China, Collaborative Innovation Center for Cancer Medicine, Guangdong Key Laboratory of Nasopharyngeal Carcinoma Diagnosis and Therapy, Guangzhou, Guangdong Province, People's Republic of China \\ ${ }^{2}$ Department of Nasopharyngeal Carcinoma, Sun Yat-sen University Cancer Center, Guangzhou, Guangdong Province, People's Republic of China \\ ${ }^{3}$ Department of Pathology, Sun Yat-Sen Memorial Hospital, Guangzhou, Guangdong Province, People's Republic of China \\ ${ }^{4}$ Department of Pathology, Sun Yat-sen University Cancer Center, Guangzhou, Guangdong Province, People's Republic of China}

Acknowledgements We thank Dr Lei Jiao from Panovue (Beijing, China) for technical help with the multispectral analysis.

Contributors H-QM, L-QT, and J-ML designed the study. S-LL, L-JB, X-SS, B-BX, $X-Y L, J-J Y$, and Z-JL collected the data. L-JB, S-MY, S-LL, D-HL, RS constructed the TMAs. Z-XL, S-LL, L-JB, X-SS, X-YL and B-BX analyzed and interpreted the data. S-LL, L-JB, X-SS, X-YL, and B-BX wrote the manuscript. H-QM, L-QT, J-ML, Z-XL, $D-H L, R S$, and $Q-Y C$ revised the manuscript. S-LL, Z-XL, and X-SS performed the statistical analysis. All authors reviewed the manuscript and approved the final version.

Funding This work was supported by grants from the National Key R\&D Program of China (2017YFC1309003, 2017YFC0908500), the National Natural Science Foundation of China (No 81425018, № 81672868, № 81802775), the Sci-Tech Project Foundation of Guangzhou City (201707020039), the Sun Yat-sen University Clinical Research 5010 Program, the Special Support Plan of Guangdong Province
(No 2014TX01R145), the Natural Science Foundation of Guangdong Province (No 2017A030312003, № 2018A0303131004), the Natural Science Foundation of Guangdong Province for Distinguished Young Scholar (№ 2018B030306001), the Sci-Tech Project Foundation of Guangdong Province (No 2014A020212103), the Health \& Medical Collaborative Innovation Project of Guangzhou City (No 201400000001, No 201803040003), Pearl River S\&T Nova Program of Guangzhou (No 201806010135), the Planned Science and Technology Project of Guangdong Province (2019B020230002), the National Science \& Technology Pillar Program during the Twelfth Five-year Plan Period (No 2014BAI09B10), and the Fundamental Research Funds for the Central Universities.

Competing interests None declared.

Patient consent for publication Obtained.

Ethics approval The study was performed with the approval of the Institutional Review Board (IRB) of Sun Yat-Sen University Cancer Center.

Provenance and peer review Not commissioned; externally peer reviewed.

Data availability statement Data and material presented in this study are available on request.

Open access This is an open access article distributed in accordance with the Creative Commons Attribution Non Commercial (CC BY-NC 4.0) license, which permits others to distribute, remix, adapt, build upon this work non-commercially, and license their derivative works on different terms, provided the original work is properly cited, appropriate credit is given, any changes made indicated, and the use is non-commercial. See http://creativecommons.org/licenses/by-nc/4.0/.

ORCID iD

Hai-Qiang Mai http://orcid.org/0000-0003-0214-203X

\section{REFERENCES}

1 Torre LA, Bray F, Siegel RL, et al. Global cancer statistics, 2012. CA Cancer J Clin 2015;65:87-108.

2 Wee JTS, Ha TC, Loong SLE, et al. Is nasopharyngeal cancer really a "Cantonese cancer"? Chin J Cancer 2010;29:517-26.

3 Pan JJ, Ng WT, Zong JF, et al. Prognostic nomogram for refining the prognostication of the proposed 8th edition of the AJCC/UICC staging system for nasopharyngeal cancer in the era of intensitymodulated radiotherapy. Cancer 2016;122:3307-15.

4 Lai S-Z, Li W-F, Chen L, et al. How does intensity-modulated radiotherapy versus conventional two-dimensional radiotherapy influence the treatment results in nasopharyngeal carcinoma patients? Int J Radiat Oncol Biol Phys 2011;80:661-8.

5 Pagès $F$, Mlecnik $B$, Marliot $F$, et al. International validation of the consensus immunoscore for the classification of colon cancer: a prognostic and accuracy study. Lancet 2018;391:2128-39.

6 Fridman $\mathrm{WH}$, Pagès $\mathrm{F}$, Sautès-Fridman $\mathrm{C}$, et al. The immune contexture in human tumours: impact on clinical outcome. Nat Rev Cancer 2012;12:298-306.

7 Galon J, Angell HK, Bedognetti D, et al. The continuum of cancer immunosurveillance: prognostic, predictive, and mechanistic signatures. Immunity 2013;39:11-26.

8 Jain A, Chia WK, Toh HC. Immunotherapy for nasopharyngeal cancer-a review. Chin Clin Oncol 2016;5:22.

$9 \mathrm{Yu}$ Y, Ke L, Lv X, et al. The prognostic significance of carcinomaassociated fibroblasts and tumor-associated macrophages in nasopharyngeal carcinoma. Cancer Manag Res 2018;10:1935-46.

10 Chen X, Li X, Zhao F, et al. [Distribution and prognostic significance of tumor-infiltrating mast cells in nasopharyngeal carcinoma]. Zhonghua Er Bi Yan Hou Tou Jing Wai Ke Za Zhi 2015;50:306-11.

11 Li Y-W, Qiu S-J, Fan J, et al. Intratumoral neutrophils: a poor prognostic factor for hepatocellular carcinoma following resection. $J$ Hepatol 2011;54:497-505.

12 Luen SJ, Salgado R, Fox S, et al. Tumour-Infiltrating lymphocytes in advanced HER2-positive breast cancer treated with pertuzumab or placebo in addition to trastuzumab and docetaxel: a retrospective analysis of the CLEOPATRA study. Lancet Oncol 2017;18:52-62.

13 Wang Y-Q, Chen Y-P, Zhang Y, et al. Prognostic significance of tumor-infiltrating lymphocytes in nondisseminated nasopharyngeal carcinoma: a large-scale cohort study. Int J Cancer 2018;142:2558-66.

14 Freeman GJ, Long AJ, Iwai Y, et al. Engagement of the PD-1 immunoinhibitory receptor by a novel B7 family member leads to negative regulation of lymphocyte activation. J Exp Med 2000;192:1027-34. 
15 Boenisch O, D'Addio F, Watanabe T, et al. TIM-3: a novel regulatory molecule of alloimmune activation. J Immunol 2010;185:5806-19.

16 Wang J, Sanmamed MF, Datar I, et al. Fibrinogen-Like protein 1 is a major immune inhibitory ligand of LAG-3. Cell 2019;176:e12:334-47.

17 Gandhi MK, Moll G, Smith C, et al. Galectin-1 mediated suppression of Epstein-Barr virus specific T-cell immunity in classic Hodgkin lymphoma. Blood 2007;110:1326-9.

18 Zhu Q, Cai M-Y, Chen C-L, et al. Tumor cells PD-L1 expression as a favorable prognosis factor in nasopharyngeal carcinoma patients with pre-existing intratumor-infiltrating lymphocytes. Oncoimmunology 2017;6:e1312240.

19 Ono T, Azuma K, Kawahara A, et al. Prognostic stratification of patients with nasopharyngeal carcinoma based on tumor immune microenvironment. Head Neck 2018;40:2007-19.

20 Turley SJ, Cremasco V, Astarita JL. Immunological hallmarks of stromal cells in the tumour microenvironment. Nat Rev Immunol 2015;15:669-82.

21 Tumeh PC, Harview CL, Yearley JH, et al. Pd-1 blockade induces responses by inhibiting adaptive immune resistance. Nature 2014;515:568-71.

22 Carey CD, Gusenleitner D, Lipschitz M, et al. Topological analysis reveals a PD-L1-associated microenvironmental niche for ReedSternberg cells in Hodgkin lymphoma. Blood 2017;130:2420-30.

23 Jiang F, Yu W, Zeng F, et al. Pd-1 high expression predicts lower local disease control in stage IV MO nasopharyngeal carcinoma. BMC Cancer 2019;19:503.

24 Anderson AC. TIM-3: an emerging target in the cancer immunotherapy landscape. Cancer Immunol Res 2014;2:393-8.

25 Nguyen LT, Ohashi PS. Clinical blockade of PD1 and LAG3--potentia mechanisms of action. Nat Rev Immunol 2015;15:45-56.

26 Miksch RC, Schoenberg MB, Weniger M, et al. Prognostic impact of tumor-infiltrating lymphocytes and neutrophils on survival of patients with upfront resection of pancreatic cancer. Cancers 2019;11:39.

27 Tanis E, Julié C, Emile J-F, et al. Prognostic impact of immune response in resectable colorectal liver metastases treated by surgery alone or surgery with perioperative FOLFOX in the randomised EORTC study 40983. Eur J Cancer 2015;51:2708-17.

28 Zhang J, Fang W, Qin T, et al. Co-Expression of PD-1 and PD-L1 predicts poor outcome in nasopharyngeal carcinoma. Med Oncol 2015;32:86.

29 Ooft ML, van Ipenburg JA, Sanders ME, et al. Prognostic role of tumour-associated macrophages and regulatory T cells in EBVpositive and EBV-negative nasopharyngeal carcinoma. J Clin Pathol 2018;71:267-74.

30 Chen T-C, Chen C-H, Wang C-P, et al. The immunologic advantage of recurrent nasopharyngeal carcinoma from the viewpoint of Galectin-9/Tim-3-related changes in the tumour microenvironment. Sci Rep 2017;7:10349.

31 Budwit-Novotny DA, McCarty KS, Cox EB, et al. Immunohistochemical analyses of estrogen receptor in endometrial adenocarcinoma using a monoclonal antibody. Cancer Res 1986;46:5419-25.

32 Camp RL, Dolled-Filhart M, Rimm DL. X-tile: a new bio-informatics tool for biomarker assessment and outcome-based cut-point optimization. Clin Cancer Res 2004;10:7252-9.

33 Lin J-C, Wang W-Y, Chen KY, et al. Quantification of plasma EpsteinBarr virus DNA in patients with advanced nasopharyngeal carcinoma. N Engl J Med 2004;350:2461-70.

34 Leung S-fai, Zee B, Ma BB, et al. Plasma Epstein-Barr viral deoxyribonucleic acid quantitation complements tumor-nodemetastasis staging prognostication in nasopharyngeal carcinoma. $J$ Clin Oncol 2006;24:5414-8.
35 Fang W, Zhang J, Hong S, et al. EBV-driven LMP1 and IFN- $\gamma$ up-regulate PD-L1 in nasopharyngeal carcinoma: implications for oncotargeted therapy. Oncotarget 2014;5:12189-202.

36 Chan OSH, Kowanetz M, Ng WT, et al. Characterization of PD-L1 expression and immune cell infiltration in nasopharyngeal cancer. Oral Oncol 2017;67:52-60.

37 Kluger HM, Zito CR, Turcu G, et al. Pd-L1 studies across tumor types, its differential expression and predictive value in patients treated with immune checkpoint inhibitors. Clin Cancer Res 2017;23:4270-9.

38 Xu-Monette ZY, Xiao M, Au Q, et al. Immune profiling and quantitative analysis decipher the clinical role of Immune-Checkpoint expression in the tumor immune microenvironment of DLBCL. Cancer Immunol Res 2019;7:644-57.

39 Hodi FS, O'Day SJ, McDermott DF, et al. Improved survival with ipilimumab in patients with metastatic melanoma. N Engl $\mathrm{J} \mathrm{Med}$ 2010;363:711-23.

40 Powles T, Eder JP, Fine GD, et al. MPDL3280A (anti-PD-L1) treatment leads to clinical activity in metastatic bladder cancer. Nature 2014;515:558-62.

41 Hamid O, Robert C, Daud A, et al. Safety and tumor responses with lambrolizumab (anti-PD-1) in melanoma. N Engl J Med 2013;369:134-44.

42 Ma BBY, Lim W-T, Goh B-C, Hui EP, et al. Antitumor activity of nivolumab in recurrent and metastatic nasopharyngeal carcinoma: an international, multicenter study of the Mayo clinic phase 2 Consortium (NCl-9742). J Clin Oncol 2018;36:1412-8.

43 Hsu C, Lee S-H, Ejadi S, et al. Safety and antitumor activity of pembrolizumab in patients with programmed death-ligand 1-positive nasopharyngeal carcinoma: results of the KEYNOTE-028 study. $J$ Clin Oncol 2017:35:4050-6.

44 Fang W, Yang Y, Ma Y, et al. Camrelizumab (SHR-1210) alone or in combination with gemcitabine plus cisplatin for nasopharyngeal carcinoma: results from two single-arm, phase 1 trials. Lancet Oncol 2018;19:1338-50.

45 Herbst RS, Soria J-C, Kowanetz M, et al. Predictive correlates of response to the anti-PD-L1 antibody MPDL3280A in cancer patients. Nature 2014;515:563-7

46 Vilain RE, Menzies AM, Wilmott JS, et al. Dynamic changes in PD-L1 expression and immune infiltrates early during treatment predict response to PD-1 blockade in melanoma. Clin Cancer Res 2017;23:5024-33.

47 Watanabe H, Ohashi K, Nishii K, et al. A long-term response to nivolumab in a case of PD-L1-negative lung adenocarcinoma with an $<$ i $>$ EGFR $</$ i $>$ mutation and surrounding PD-L1-positive tumorassociated macrophages. Intern Med 2019;58:3033-7.

48 Ribatti D. Mast cells as therapeutic target in cancer. Eur J Pharmacol 2016;778:152-7.

$49 \mathrm{Fu} \mathrm{H}$, Zhu Y, Wang Y, et al. Identification and validation of stromal immunotype predict survival and benefit from adjuvant chemotherapy in patients with muscle-invasive bladder cancer. Clin Cancer Res 2018;24:3069-78.

50 Zheng Z, Cai Y, Chen H, et al. CXCL13/CXCR5 axis predicts poor prognosis and promotes progression through PI3K/Akt/mTOR pathway in clear cell renal cell carcinoma. Front Oncol 2018;8:682.

51 Zhu Z, Zhang X, Guo H, et al. CXCL13-CXCR5 axis promotes the growth and invasion of colon cancer cells via PI3K/Akt pathway. Mol Cell Biochem 2015;400:287-95.

52 Larbcharoensub N, Mahaprom K, Jiarpinitnun C, et al. Characterization of PD-L1 and PD-1 expression and CD8+ tumor-infiltrating lymphocyte in Epstein-Barr virus-associated nasopharyngeal carcinoma. Am J Clin Oncol 2018;41:1204-10. 